\title{
Combining Quantitative Susceptibility Mapping with Automatic Zero Reference (QSM0) and Myelin Water Fraction Imaging to Quantify Iron-Related Myelin Damage in Chronic Active MS Lesions
}

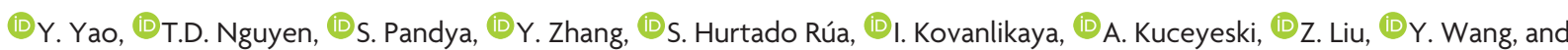 \\ (1)S.A. Gauthier \\ O-
}

\begin{abstract}
BACKGROUND AND PURPOSE: A hyperintense rim on susceptibility in chronic MS lesions is consistent with iron deposition, and the purpose of this study was to quantify iron-related myelin damage within these lesions as compared with those without rim.
\end{abstract}

MATERIALS AND METHODS: Forty-six patients had 2 longitudinal quantitative susceptibility mapping with automatic zero reference scans with a mean interval of $28.9 \pm 11.4$ months. Myelin water fraction mapping by using fast acquisition with spiral trajectory and $\mathrm{T} 2 \mathrm{prep}$ was obtained at the second time point to measure myelin damage. Mixed-effects models were used to assess lesion quantitative susceptibility mapping and myelin water fraction values.

RESULTS: Quantitative susceptibility mapping scans were on average 6.8 parts per billion higher in 116 rim-positive lesions compared with 441 rim-negative lesions $(P<.001)$. All rim-positive lesions retained a hyperintense rim over time, with increasing quantitative susceptibility mapping values of both the rim and core regions $(P<.001)$. Quantitative susceptibility mapping scans and myelin water fraction in rim-positive lesions decreased from rim to core, which is consistent with rim iron deposition. Whole lesion myelin water fractions for rim-positive and rim-negative lesions were $0.055 \pm 0.07$ and $0.066 \pm 0.04$, respectively. In the mixed-effects model, rim-positive lesions had on average 0.01 lower myelin water fraction compared with rim-negative lesions $(P<.001)$. The volume of the rim at the initial quantitative susceptibility mapping scan was negatively associated with follow-up myelin water fraction $(P<.01)$.

CONCLUSIONS: Quantitative susceptibility mapping rim-positive lesions maintained a hyperintense rim, increased in susceptibility, and had more myelin damage compared with rim-negative lesions. Our results are consistent with the identification of chronic active MS lesions and may provide a target for therapeutic interventions to reduce myelin damage.

ABBREVIATIONS: FAST-T2 = fast acquisition with spiral trajectory and T2-prep; GRE = gradient-echo; MWF = myelin water fraction; ppb = parts per billion; $\mathrm{QSM}=$ quantitative susceptibility mapping; $\mathrm{QSMO}=\mathrm{QSM}$ with automatic uniform CSF zero reference; rim $-=$ rim-negative; rim + = rim-positive

M $S$ is an inflammatory demyelinating and neurodegenerative disease of the CNS. Differentiating MS lesions, especially chronic lesions, may provide a biomarker for disease progression and a therapeutic target to reduce ongoing tissue damage. A subset of chronic lesions, identified as chronic active or slowly expanding lesions, have been described as having a rim of iron-enriched proinflammatory activated microglia and macrophages. ${ }^{1-4}$ Iron-enriched

Received June 16, 2017; accepted after revision October 13.

From the Department of Radiology (Y.Y., Y.Z.), Tongji Hospital, Tongji Medical College, Huazhong University of Science \& Technology, Wuhan, China; Departments of Radiology (Y.Y., T.D.N., S.P., I.K., A.K., Z.L., Y.W.) and Neurology (S.A.G.), Weill Cornell Medicine, New York, New York; Department of Mathematics (S.H.R.), Cleveland State University, Cleveland, Ohio; and Department of Biomedical Engineering (Z.L., Y.W.), Cornell University, Ithaca, New York.

This work was supported by grant RG 4661-A-2 from the National Multiple Sclerosis Society, grant RO1 NS090464 from the National Institutes of Health, and grant UL1 TR000456-06 from the Weill Cornell Clinical and Translational Science Center (CTSC).

Paper previously presented at: Annual Meeting and Exhibition of the International Society for Magnetic Resonance in Medicine, April 22-27, 2017; Honolulu, Hawaii. proinflammatory microglia release cytotoxins to adjacent oligodendrocytes, ${ }^{5}$ limit remyelination, and contribute to further demyelination in chronic active lesions. ${ }^{3}$ Therefore, identifying MS lesions with iron accumulation may enable the prediction of tissue damage.

MR imaging with a gradient-echo (GRE) sequence is sensitive to iron ${ }^{1,6}$ and has been explored by many investigators to detect an iron rim in chronic active MS lesions. ${ }^{1,3,4,7-9}$ Iron may be detected as hypointensity on a $\mathrm{T}^{*}$-weighted GRE magnitude image or its phase-enhanced version known as SWI. ${ }^{8,10}$ An $2^{*}(=1 \div$ $\mathrm{T} 2{ }^{*}$ ) map computed from a multiecho GRE magnitude image can be used to estimate iron content. ${ }^{11}$ Unfortunately, precise mapping of iron location by these magnitude-based approaches is

Please address correspondence to Susan A. Gauthier, DO, MPH, Department of Neurology, Weill Cornell Medical College, 1305 York Ave, Suite Y217, New York, NY 10021; e-mail: sag2015@med.cornell.edu

- Indicates open access to non-subscribers at www.ajnr.org

Indicates article with supplemental on-line photos.

http://dx.doi.org/10.3174/ajnr.A5482 
hindered by blooming artifacts, particularly at high iron concentrations. ${ }^{12}$ Alternatively, the local magnetic field derived from a GRE phase image has been studied to quantify iron. ${ }^{13,14}$ However, because the local phase is affected by magnetic sources in the surrounding tissue, the phase pattern may not represent the true magnetic susceptibility pattern. ${ }^{15}$ Quantitative susceptibility mapping $(\mathrm{QSM})^{16}$ is a phase-based magnetic field deconvolution technique that overcomes the blooming artifacts and provides more accurate quantification and localization of the magnetic sources. ${ }^{17,18}$ QSM has been established as a more sensitive and quantitative technique for measuring brain iron compared with $\mathrm{T} 2{ }^{*}, \mathrm{R} 2$, and R2 ${ }^{*}{ }^{19,20}$ Studies have emerged combining QSM with additional MR tissue parameters, such as $\mathrm{R} 2^{*}$, to characterize the pattern of iron deposition and demyelination among various MS lesions. ${ }^{21-23}$ However, the interpretation of iron accumulation and the associated tissue damage can be complicated because QSM and R2 ${ }^{*}$ are both sensitive to iron and myelin. Moreover, accurate tracking of lesion susceptibility changes over time is hindered by the lack of a reliable susceptibility reference, given that CSF can often appear highly heterogeneous on QSM and normalappearing white matter may undergo pathologic iron or myelin changes. $^{14}$

This study aimed to address these challenges by combining 2 recently developed techniques: myelin water fraction (MWF) imaging by using fast acquisition with spiral trajectory and T2-prep $(\text { FAST-T2 })^{24,25}$ and QSM with automatic uniform CSF zero reference (QSM0). MWF is a well-validated quantitative MR imaging biomarker for myelin, ${ }^{26,27}$ which can be mapped efficiently and reproducibly with FAST-T2. ${ }^{25,28}$ The QSM0 algorithm improves QSM zero reference selection by enforcing the susceptibility homogeneity of CSF within the brain ventricles and eliminating the need for manual drawing of CSF ROIs. QSM0 and MWF were used to identify MS lesions with a hyperintense rim pattern consistent with iron deposition and to assess the extent of myelin damage found within these lesions.

\section{MATERIALS AND METHODS \\ Patient Population}

This was a retrospective study of a cohort of 46 patients with MS (14 men, 32 women; mean age, $43.6 \pm 10.7$ years) selected from a prospective, ongoing clinical MS MR imaging data base from October 2011 to April 2015. The only inclusion criteria consisted of having simultaneous QSM and FAST-T2 sequences and a prior QSM. This cohort consisted of 1 patient with clinically isolated syndrome, 44 with relapsing-remitting MS, and 1 with secondary-progressive MS (mean disease duration, $8.7 \pm 7.5$ years; mean Expanded Disability Status Scale score, $1.78 \pm 1.84$ ). Forty-three patients were on various disease-modifying therapies, and 3 were untreated. Patients identified for analysis had completed 2 longitudinal brain MR imaging examinations with a mean time interval of $28.9 \pm 11.4$ months. Approximately $45 \%$ of the patients changed to a different MS treatment between the 2 MR imaging time points. This study was approved by the Weill Cornell Medicine institutional review board.

\section{MR Imaging Data Acquisition}

Brain MR imaging was performed on a 3T MR scanner (Signa HDxt; GE Healthcare, Milwaukee, Wisconsin) with an 8-channel head coil. The scanning protocol consisted of standard T1weighted and T2-weighted sequences for anatomy and multiecho GRE imaging for QSM, as well as gadolinium-enhanced T1weighted imaging to detect blood-brain barrier disruption. In addition, a FAST-T2 sequence was run at the second time point to map lesion MWF as a quantitative biomarker of myelin damage. The typical imaging parameters for pertinent imaging sequences were as follows: 1) T2-weighted multisection 2D fast spin-echo: TR, $5250 \mathrm{~ms}$; TE, $86 \mathrm{~ms}$; axial field of view, $24 \mathrm{~cm}$; phase field of view factor, 0.75 ; acquisition matrix, $416 \times 256$ interpolated to $512 \times 512$; section thickness, $3 \mathrm{~mm}$ without gap; flip angle, $90^{\circ}$; echo-train length, 23; number of signal averages, 2 ; and readout bandwidth, $\pm 50 \mathrm{kHz}$; 2) multiecho GRE: TR, $57 \mathrm{~ms}$; first TE, 4.3 $\mathrm{ms}$; echo spacing, $4.8 \mathrm{~ms}$; echo-train length, 11; axial field of view, $24 \mathrm{~cm}$; phase field of view factor, 0.8 ; acquisition matrix, $416 \times$ 320 interpolated to $512 \times 512$; section thickness, $3 \mathrm{~mm}$; flip angle, $20^{\circ}$; bandwidth, $244 \mathrm{kHz}$; number of signal averages, 0.75 ; and readout bandwidth, $\pm 62.5 \mathrm{kHz} ; 3$ ) 3D stack-of-spirals FAST-T2: spiral TR, $7.8 \mathrm{~ms}$; spiral TE, $0.5 \mathrm{~ms}$; nominal T2-prep times, $0 \mathrm{~ms}$ (T2-prep turned off), 7.6, 17.6, 27.6, 67.6, 147.6, and $307.6 \mathrm{~ms}$; number of spiral leaves per stack, 32; axial field of view, $24 \mathrm{~cm}$; acquisition matrix, $192 \times 192$ interpolated to $256 \times 256$; section thickness, $5 \mathrm{~mm}$; number of sections, 32; flip angle, $10^{\circ}$; and readout bandwidth, $\pm 125 \mathrm{kHz}$.

\section{MR Imaging Postprocessing}

Brain QSM0 maps were reconstructed from multiecho GRE data by using the morphology-enabled dipole inversion method ${ }^{29,30}$ and incorporating automated segmentation and regularization specific to CSF. Briefly, CSF within the lateral ventricles was identified by thresholding of the $\mathrm{R} 2^{*}$ map $\left(\mathrm{R} 2^{*}<5\right.$ seconds $\left.^{-1}\right)$ and imposing voxel connectivity. A regularization term penalizing susceptibility variation within the CSF mask was incorporated into the morphology-enabled dipole inversion algorithm to search for a solution with homogeneous CSF susceptibility. MWF maps were reconstructed from FAST-T2 data by using a multivoxel nonlinear least-squares data-fitting algorithm with spatial smoothness constraints. ${ }^{25}$ The lower and upper T2 bounds for each of the 3 water pools (in milliseconds) were set to [5 20], [20 200], and [200 2000], respectively (corresponding to myelin water, intra- and extracellular water, and long-T2 water such as CSF). MWF was calculated as the ratio of the myelin water signal and the total water signal within a voxel. Anatomic images and MWF maps were coregistered to GRE magnitude images (and the associated QSM maps) by using the FMRIB Linear Image Registration Tool (FLIRT; https://fsl.fmrib.ox.ac.uk/fsl/fslwiki/FLIRT) algorithm. ${ }^{31}$

\section{Image Analysis}

Two neuroradiologists (Y.Z. and Y.Y) with 6 and 4 years of experience, respectively, independently reviewed all images. First, MS lesions were identified and manually traced on T2-weighted images. Only gadolinium-negative lesions were considered in the primary analysis. Lesions were identified as rim-positive ( $\mathrm{rim}+$ ) or rimnegative (rim-) based on the consensus of both reviewers regarding 

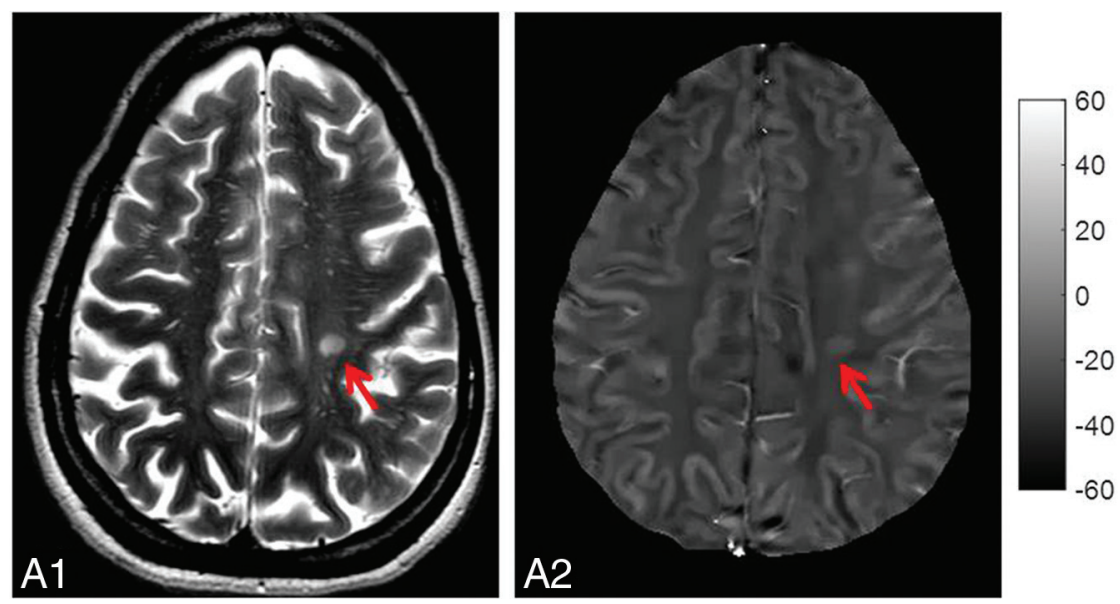

clusion. The mean QSM and MWF values within the lesion core and rim areas of rim+ lesions were compared by a paired $t$ test.

\section{RESULTS}

Identification of QSM Rim + and Rim-Lesions

All T2-weighted hyperintense lesions that were present at both time points were reviewed and considered chronic if they were not enhancing with gadolinium. Two lesions were excluded because of a subthreshold volume $\left(<14 \mathrm{~mm}^{3}\right)$, and 15 lesions were excluded because of image artifacts and poor QSM quality. The remaining lesions included 116 rim + lesions from 35 of 46 patients with MS (34 relapsing-remitting and 1 secondary-progressive) and $441 \mathrm{rim}-\mathrm{le}$ sions from 42 of 46 patients with MS (1 clinically isolated syndrome, 40 relapsing-remitting, and 1 secondary-progressive). Most patients (31 of 46) were found to have both rim + and rim- lesions. Of the patients having 1 lesion subtype, 4 of 15 had only rim + lesions and the remaining patients (11 of 15 ) had only rim - lesions. Ten patients had gadolinium-enhancing lesions at any time point (12 lesions). The clinical characteristics (ie, disease duration or Expanded Disability Status Scale score) between the 2 groups of patients having only

1. Top panel, Example of rim - lesion (red arrow): T2-weighted image (A7) and QSM (A2) patient with relapsing-remitting MS. Bottom panel, Example of rim + lesion (yellow arrow): T2weighted image (BI) and QSM (B2) in another patient with relapsing-remitting MS.

their visual appearance on QSM obtained at each time point (QSM1 and QSM2). In the case of a disagreement, a third neuroradiologist (I.K.) with 20 years of experience was called on to determine the lesion subtype. Next, ROI analysis was performed by using ITKSNAP software (version 3.2; http://www.itksnap.org/) to obtain regional QSM measurements within the identified lesions. ROIs were traced on QSM and MWF for the whole lesion by using the T2weighted lesion ROI as a starting point, with additional manual adjustments if needed. Central veins, identified as vessel-like structures with a hyperintense QSM appearance, were manually removed from QSM ROIs. In addition, for rim + lesions, the lesion core (defined as the part of the lesion that extends from the center to the inner boundary of the hyperintense QSM rim) was traced on QSM and then transferred to MWF maps and manually edited if necessary. Lesion rim ROI was defined as the ROI difference between the whole lesion and the lesion core.

\section{Statistical Analysis}

Mixed-effects models were implemented to assess the variables of interest (lesion QSM and MWF values) among rim + and rim - lesions. The modeling strategy accounts for multiple lesions per patient and repeated measurements (longitudinal analysis), and the following covariates were always considered: patient age, sex, T2-weighted lesion volume, and time interval between MRIs. The final model is reported after using a back-fitting procedure set at $\alpha=0.10$ for in-
1 lesion subtype were similar. Among all the patients, there was no significant correlation between the number of rim + lesions and disease duration $(P=.43)$.

\section{Comparison of QSM Rim + and Rim - Lesions}

Figure 1 shows an example of T2-weighted images and corresponding QSM from 2 patients with relapsing-remitting MS, illustrating the appearance of rim + and rim- lesions on QSM (4 additional patient examples are shown in On-line Fig 1). For whole lesion ROI, QSM1 of rim + lesions and rim- lesions were $6.0 \pm 14.4$ parts per billion (ppb) and $-7.0 \pm 17.1 \mathrm{ppb}$, respectively (On-line Fig 2). There was no significant difference between the patient age among the rim + and rim - lesion subgroups $(44.1 \pm 10.6$ versus $43.5 \pm 10.7 ; P=.82)$, and the Expanded Disability Status Scale score was similar among the lesion subtype groups $(P=.80)$. After accounting for patient variability as a random effect (mixed-effects model), the QSM1 values in rim+ lesions were on average $6.8 \mathrm{ppb}$ higher than those in rim- lesions $(P<.001)$. There was no significant association found with T2weighted lesion volume, sex, or patient age.

\section{Longitudinal Assessment of QSM Classification}

QSM classification at follow-up MR imaging predominantly remained the same. All rim + lesions retained the hyperintense rim 

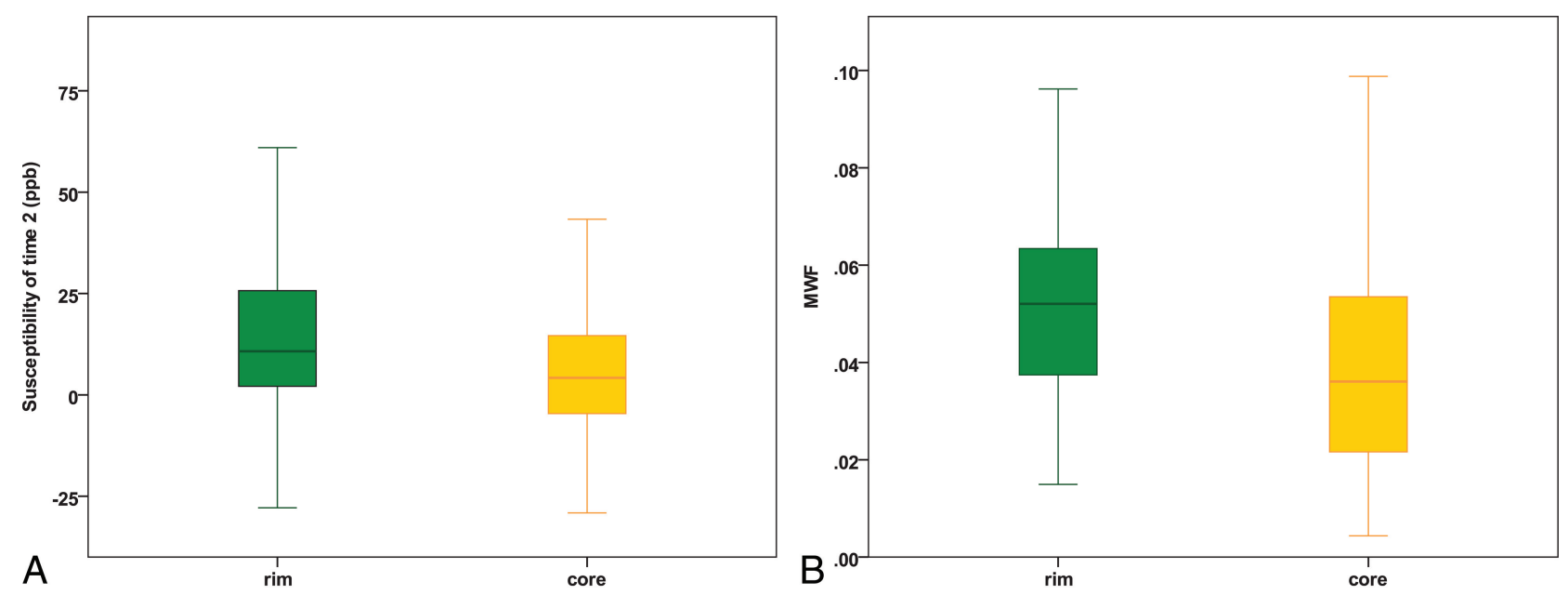

FIG 2. Comparison of susceptibility $(A)$ and MWF (B) differences within the rim and core of QSM hyperintense rim + lesions. Rim ROIs (green) have significantly higher susceptibility and higher MWF compared with core ROls (yellow).

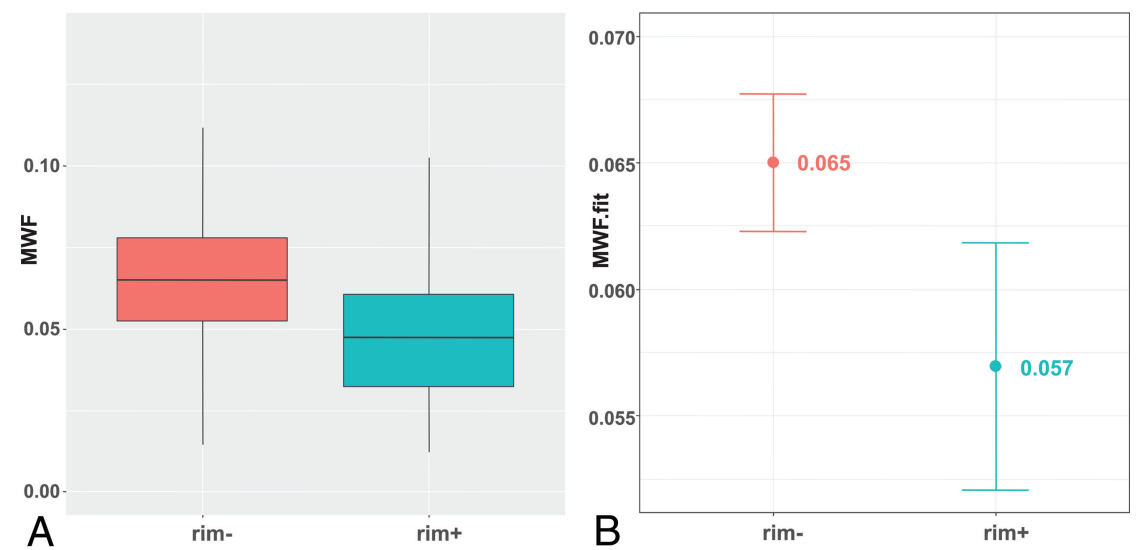

FIG 3. A, Boxplot of rim - (pink) and rim + (blue) lesions, which demonstrates lower MWF in rim + compared with rim - lesions. B, Confidence interval plot of rim - (pink) and rim + (blue) lesions derived from a mixed-effects model, which demonstrates lower MWF mean effect in rim + lesions compared with rim - lesions after controlling for multiple lesions per patient and T2 lesion size $(P<.001)$.

at both time points (QSM1 and QSM2). Three lesions were initially identified as rim- at QSM1 and demonstrated a positive hyperintense rim at QSM2. T2 lesion volume remained stable between the 2 longitudinal MR imagings for both $\operatorname{rim}+(P=.94)$ and $\operatorname{rim}-(P=.07)$ lesions. Rim + lesions demonstrated an increase in whole-lesion QSM $(4.8 \pm 10.3 \mathrm{ppb})$ over time, with both the core and rim ROI values increasing by $4.0 \pm 11.6 \mathrm{ppb}$ and $5.3 \pm 11.0 \mathrm{ppb}$, respectively. QSM values for rim-lesions also increased over time, but with smaller differences $(2.3 \pm 10.8 \mathrm{ppb})$. A mixed-effects model confirmed a significant change in QSM values within the rim $(P<.001)$ and core $(P<.001)$ regions of rim+ lesions.

\section{QSM and MWF Assessment of Lesions}

At the second time point, QSM and MWF values within the rim and core regions of rim + lesions were examined to support the premise that iron is present at the lesion edge. The volumes of the rim and core area were $271.4 \pm 200.9 \mathrm{~mm}^{3}$ and $116.6 \pm 113.7 \mathrm{~mm}^{3}$, respectively. There was a centripetal pattern of reduction observed in both QSM and MWF. In rim + lesions, the mean QSM value significantly decreased from the $\operatorname{rim}(13.3 \pm 16.3 \mathrm{ppb})$ to the core
(5.9 $\pm 15.2 \mathrm{ppb} ; P<.01 ;$ Fig 2$)$. As with QSM, the rim + lesions demonstrated a significant decrease of MWF from the $\operatorname{rim}(0.052 \pm 0.022)$ to the core $(0.039 \pm$ $0.022 ; P<.01$; Fig 2$)$.

MWF whole-lesion values for rim + lesions were lower compared with rimlesions $(0.055 \pm 0.070$ and $0.066 \pm$ 0.040 , respectively; Fig. 3) and found to be consistently lower with the exclusion of patients with gadolinium-enhancing lesions $(0.044 \pm 0.021$ and $0.068 \pm$ 0.040 , respectively; On-line Fig 3 ). This difference became more obvious in the mixed-effects model, which showed that whole-lesion MWF in rim + lesions was on average 0.01 (Fig 3) lower compared with rim $-(P<.001)$. A similar difference in MWF $(0.011 ; P<.001)$ was found with the exclusion of patients with gadolinium-enhancing lesions (On-line Fig 3). T2-weighted lesion volume remained a significant covariate in the final model $(P<.001)$. For every cubic millimeter increase in T2-weighted volume, MWF decreased by 0.000013 . The MWF differences among rim + and rim - lesions can be appreciated on the MWF map shown in Fig 4. In rim+ lesions, we further expanded our analysis to explore the specific relationship of the hyperintense rim at the first time point (susceptibility value and volume on QSM1) and subsequent MWF. In the mixed-effects model, the volume of the rim at QSM1 $(P<.01)$ was the only significant covariate; for every cubic millimeter increase in QSM1 rim volume, lesion MWF decreased by 0.00002 . Figure 5 highlights the MWF differences between thick rim and thin rim lesions.

\section{DISCUSSION}

Our study is one of many demonstrating that GRE MR imaging can identify a discrete subset of chronic MS lesions, ${ }^{1,3,4,7-9}$ and as with other studies, we demonstrate the retention of the iron rim and more tissue damage in these selected lesions. There are 3 

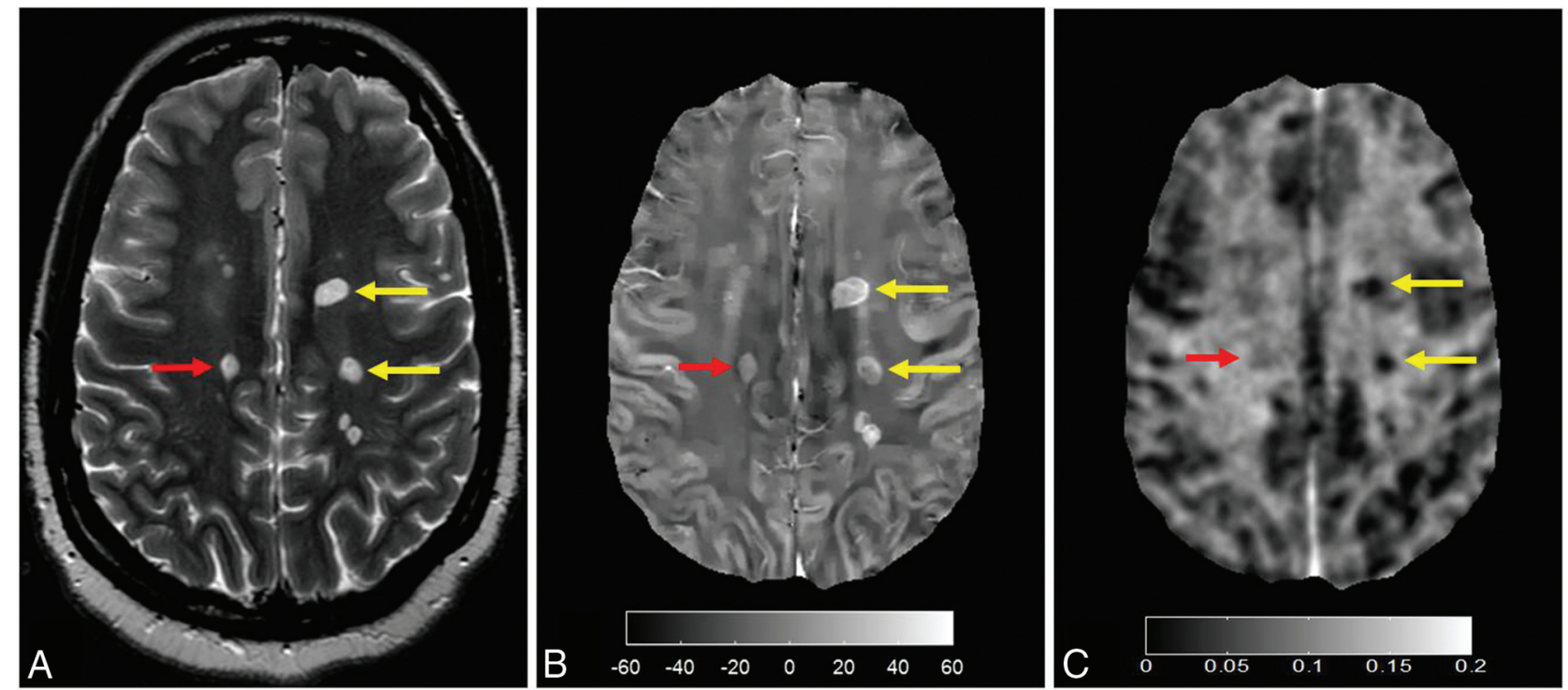

FIG 4. T2-weighted $(A), Q S M(B)$, and MWF map (C) images of a patient with relapsing-remitting MS are shown. The hypointense appearance of rim + lesions (yellow arrows) on the MWF map is consistent with a lower MWF compared with the more isointense appearance of a rim - lesion (red arrow).
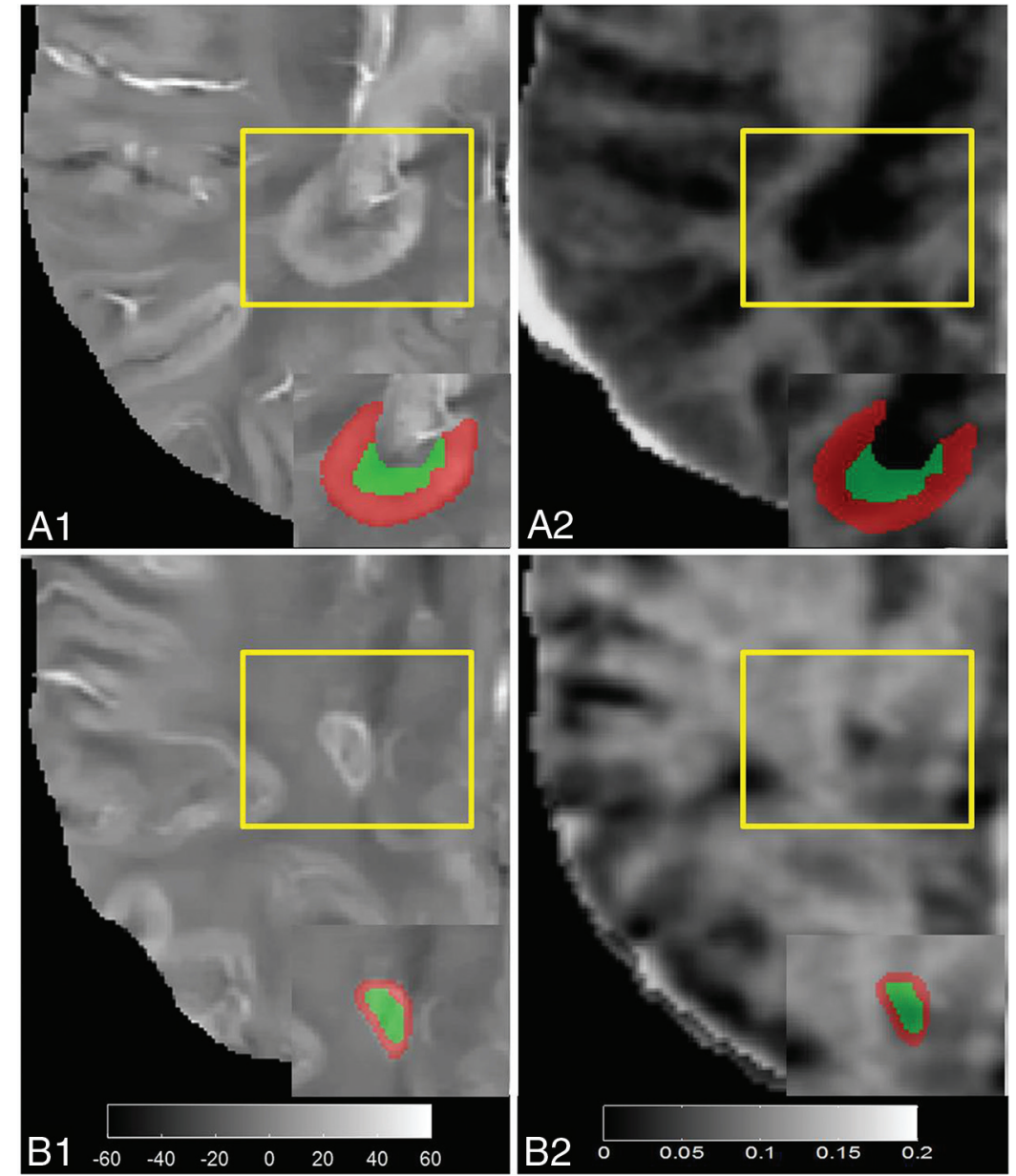

FIG 5. Top panel, An example of thick rim lesion (yellow rectangle): QSM (AT) and MWF (A2) in a patient with relapsing-remitting MS. The QSM and MWF values in the core and rim are as follows: $-5.2827 \pm 14.3945 \mathrm{ppb}$ and $2.2262 \pm 17.7374 \mathrm{ppb} ; 1.5204 \% \pm 1.3975 \%$ and $4.2542 \% \pm 2.5327 \%$. Bottom panel, An example of thin rim lesion (yellow rectangle): QSM (BT) and MWF (B2) in another patient with relapsing-remitting MS. The QSM and MWF values in the core and rim are as follows: $-3.0355 \pm 10.271$ $\mathrm{ppb}$ and $0.9208 \pm 13.5405 \mathrm{ppb} ; 7.4161 \% \pm 1.5726 \%$ and $8.4678 \% \pm 1.3358 \%$. The hypointense appearance of a thick rim lesion on the MWF map is lower compared with the thin rim lesions. unique components to our study: 1) the application of QSM0 for a more accurate identification and quantification of iron rim lesions in a longitudinal study, compared with conventional QSM, SWI, or MR phase; 2) a multitechnique approach of combining QSM with a myelin-specific MR imaging acquisition (MWF); and 3) quantitatively examining the susceptibility and myelin content within the individual components of QSM hyperintense rim+ lesions (rim and core regions). Our work provides further in vivo evidence that GRE MR imaging can identify chronic lesions with more demyelination, which is consistent with the known histopathologic classification of a chronic active MS lesion.

QSM studies of MS lesions have uncovered interesting dynamics wherein lesion susceptibility substantially increases shortly after gadolinium enhancement and remains high for the first few years. ${ }^{32,33}$ The susceptibility increase in MS lesions can come from demyelination and/or iron accumulation. ${ }^{17}$ The integrated multitechnique QSM+MWF approach described in this study allows for a novel quantitative investigation of the connection between iron-associated inflammation and tissue damage in the brains of patients with MS. Recent patient studies demonstrated increased tissue damage on T1weighted images for MS lesions with a persistent rim on phase images ${ }^{4}$ and a 
higher occurrence of lesions with a QSM rim in patients with progressive MS and increasing disability. ${ }^{23}$ However, it is difficult to quantify tissue damage on T1-weighted hypointensity. In addition, studies using QSM and R2 ${ }^{*}$ described QSM hyperintense rim lesions as having minor myelin loss compared with other lesion subtypes, ${ }^{22}$ which is inconsistent with the histopathologic description of chronic active lesions as having extensive demyelination $^{34}$; this discrepancy may be the consequence of $\mathrm{R} 2^{*}$ being influenced by iron. For a quantitative assessment of tissue damage in white matter MS lesions, MWF is regarded as an indirect measure of myelin with a relatively high specificity given the strong pathologic correlation. ${ }^{26-28}$ We recognize that an increase of susceptibility within a chronic lesion can be due to demyelination or iron deposition, which limits direct iron quantification by QSM. ${ }^{17}$ We identify QSM rim + lesions as having a rim of iron based upon the susceptibility gradient between the rim and core regions. Support of this approach is based upon 3 factors: 1) a known pattern of demyelination found within chronic active MS

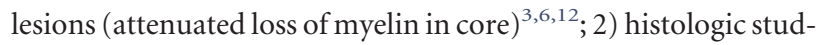
ies demonstrating that GRE can detect iron within activated microglia and macrophages at the edge of chronic active MS lesions $^{1,3,4,11,14,15}$; and 3) our own data, demonstrating a decrease in both susceptibility and MWF from rim to core, which can only be explained by iron deposition. Thus, we conclude that iron is contributing, at least in part, to the signal at the rim, but importantly, we are not quantifying the absolute extent that iron or myelin is contributing to the QSM signal.

This QSM+MWF study helps highlight the clinical importance of chronic active lesions, which have been largely ignored in current clinical practice, but may potentially be treated to reduce tissue damage and possibly slow disease progression as suggested by clinical and immunohistologic data. After the acute stage, a significant increase in both QSM and R2 ${ }^{*}$ occurs in lesions that are no longer enhancing, yet under 1 year of age, suggesting that iron release occurs during the early stages after myelin destruction. ${ }^{21}$ These findings are consistent with the release of iron secondary to myelin and oligodendrocyte destruction ${ }^{9,35}$ and provide a source for iron-laden proinflammatory microglia and for iron-driven amplification of oxidative stress within the acute MS lesion. ${ }^{2,36}$ Studies have shown evidence for oxidative stress damage to oligodendrocytes and mitochondrial and dystrophic axons in acute MS lesions, ${ }^{37}$ which consequently inhibit endogenous remyelination. Correspondingly, we found that lesions having a QSM hyperintense rim had a significantly lower MWF throughout the whole lesion compared with those identified as without rim. Interestingly, we found an association with rim volume and lesion MWF, which is consistent with thicker-rimmed chronic lesions demonstrating more active demyelination. ${ }^{34}$

Chronic active MS lesions have been found to be more prominent in progressive disease compared with the relapsing stage of the disease, and their continued expansion may play an essential role in the pathogenesis of progressive disease. ${ }^{38}$ In vivo imaging studies of phase or QSM have revealed conflicting results regarding the prevalence of rim + lesions among patients with MS. ${ }^{39}$ One previous study demonstrated a vast difference among QSM and phase results, wherein the authors concluded that QSM was superior to the depiction of spatial susceptibility patterns in MS lesions. ${ }^{15}$ Similarly, we found wide-ranging differences in lesion classification based upon QSM versus phase images (data not shown). A major contributing factor to the difficulty of identifying and quantifying rim + lesions has been the choice of susceptibility reference tissue. Wiggerman et $\mathrm{al}^{35}$ have recently demonstrated in postmortem samples that lesion phase and QSM contrast can be highly influenced by pathologic alterations in myelin and iron in normal-appearing white matter, a typical choice of QSM reference in MS lesion studies. CSF being 99\% pure water is another common choice of susceptibility reference; however, current QSM reconstruction algorithms often produce a highly inhomogeneous CSF appearance in the brain ventricles, most likely due to the susceptibility anisotropy effect of the adjacent brain white matter. Consequently, the zero reference in previous studies often depends on the manual selection of ROI, making it less suited for longitudinal studies. A unique component of our study was the use of QSM0, a recently developed QSM inversion method that automatically selects a CSF mask based on R2 ${ }^{*}$ derived from a GRE magnitude image and enforces uniformity within the CSF region. This improvement could lead to better detection and quantification of QSM lesions. Interestingly, the current study population is predominantly relapsing patients with minimal disability and differs significantly from postmortem studies, which generally lack the inclusion of younger patients. Therefore, to determine the true prevalence and incidence of these lesions among patients with MS, future studies will require a consistent imaging protocol, for which we favor QSM0, on a larger cohort of patients that includes both relapsing and progressive stages of the disease. In addition, QSM and MWF provides an opportunity to further explore the range of myelin damage of these particular lesions, especially those found in younger MS patients.

This study has several limitations. First, our analysis is limited by the incomplete longitudinal design due to MWF only being acquired at the second time point. Further studies evaluating the longitudinal relationship of QSM and MWF in acute MS lesions will improve our understanding of the complex association of lesion iron deposition and myelin damage. Although our results of combining the data from QSM and MWF provide support for iron deposition, we lack histologic validation that lesions demonstrating a hyperintense rim on QSM truly have iron deposition; however, a number of mentioned studies have validated GRE imaging to identify these lesions. Further highlighting the necessity of histopathologic validation is that paramagnetic iron is generally expected to shorten the T2 relaxation time of brain tissue, and the resulting effect on the accuracy of MWF quantification is currently not well understood. Therefore, MWF measurements in QSM rim+ lesions in the presence of elevated iron should be interpreted with caution. Last, clinical factors, such as specific treatments or medication changes, were not considered as variables of interest in our analysis. Treatment duration was the only "treatment-related" variable included in the mixed-effects models because of the complexity of individual patient management decisions. Our analysis presumes that specific MS treatments have no influence on the lesions' iron or myelin content, and we recognize that this may be a limitation; however, there is no current evidence to contradict this assumption. Furthermore, larger stud- 
ies would be required to properly assess the influence of specific treatments on QSM and MWF. In addition, in a separate analysis, we assessed the potential influence of enhancing lesions and the remote effect of inflammation related to breakdown of the bloodbrain barrier. We found that after removing patients with enhancing lesions, a significant difference in MWF remained among rim + and rim - lesions.

\section{CONCLUSIONS}

Our study demonstrated that differences exist among individual lesions based upon the QSM hyperintensity pattern. QSM rim+ lesions maintained a hyperintense rim and demonstrated an increase in susceptibility over time. Centripetal decrease in QSM and MWF identified a subset of MS lesions with excess iron deposition at the rim and more myelin damage. These results raise the possibility that QSM may provide insight into pathologic mechanisms of injury, such as iron release and chronic inflammation, within individual lesions. Furthermore, a hyperintense rim on QSM may provide a biomarker to target and study myelin injury within chronic active MS lesions. Treatments targeting the modulation of chronic CNS inflammation would provide a novel therapeutic strategy to prevent ongoing myelin damage as well as enhance remyelination and decrease disease progression.

Disclosures: Thanh Nguyen—RELATED: Grants/Grants Pending: National Institutes of Health; UNRELATED: Grants/Grants Pending: National Institutes of Health, National Multiple Sclerosis Society*. Yi Wang—RELATED: Grants/Grants Pending: National Institutes of Health, Comments: grant R01 NS090464*; UNRELATED: Grants/ Grants Pending: National Institutes of Health, Comments: we are actively seeking National Institutes of Health grants to translate our work into clinical practice*; Patents (Planned, Pending, or Issued): Cornell University, Comments: I am one of the inventors named on the QSM patents*; Stock/Stock Options: Medimagemetric, Comments: Medimagemetric is working with Cornell to commercialize QSM technology. Susan Gauthier-RELATED: Grants/Grants Pending: National Institutes of Health, Comments: grant R01NS090464, focused on multiple sclerosis lesion magnetic susceptibility activity*; UNRELATED: Grants/Grants Pending: Genzyme*, Mallinckrodt*, Novartis*. "Money paid to the institution.

\section{REFERENCES}

1. Bagnato F, Hametner S, Yao B, et al. Tracking iron in multiple sclerosis: a combined imaging and histopathological study at $7 \mathrm{Te}-$ sla. Brain 2011;134:3602-15 CrossRef Medline

2. Stephenson E, Nathoo N, Mahjoub Y, et al. Iron in multiple sclerosis: roles in neurodegeneration and repair. Nat Rev Neurol 2014;10: 459-68 CrossRef Medline

3. Dal-Bianco A, Grabner G, Kronnerwetter C, et al. Slow expansion of multiple sclerosis iron rim lesions: pathology and $7 \mathrm{~T}$ magnetic resonance imaging. Acta Neuropathol 2017;133:25-42 CrossRef Medline

4. Absinta M, Sati P, Schindler M, et al. Persistent 7-Tesla phase rim predicts poor outcome in new multiple sclerosis patient lesions. J Clin Invest 2016;126:2597-609 CrossRef Medline

5. Cairo G, Recalcati S, Mantovani A, et al. Iron trafficking and metabolism in macrophages: contribution to the polarized phenotype. Trends Immunol 2011;32:241-47 CrossRef Medline

6. Langkammer C, Schweser F, Krebs N, et al. Quantitative susceptibility mapping (QSM) as a means to measure brain iron? A post mortem validation study. Neuroimage 2012;62:1593-99 CrossRef Medline

7. Hammond KE, Metcalf M, Carvajal L, et al. Quantitative in vivo magnetic resonance imaging of multiple sclerosis at 7 Tesla with sensitivity to iron. Ann Neurol 2008;64:707-13 CrossRef Medline

8. Yao B, Ikonomidou VN, Cantor FK, et al. Heterogeneity of multiple sclerosis white matter lesions detected with $\mathrm{T} 22^{*}$-weighted imaging at 7.0 Tesla. J Neuroimaging 2015;25:799-806 CrossRef Medline

9. Mehta V, Pei W, Yang G, et al. Iron is a sensitive biomarker for inflammation in multiple sclerosis lesions. PLoS One 2013;8:e57573 CrossRef Medline

10. Haacke EM, Makki M, Ge Y, et al. Characterizing iron deposition in multiple sclerosis lesions using susceptibility weighted imaging. $J$ Magn Reson Imaging 2009;29:537-44 CrossRef Medline

11. Langkammer C, Krebs N, Goessler W, et al. Quantitative MR imaging of brain iron: a postmortem validation study. Radiology 2010; 257:455-62 CrossRef Medline

12. Li J, Chang S, Liu T, et al. Reducing the object orientation dependence of susceptibility effects in gradient echo MRI through quantitative susceptibility mapping. Magn Reson Med 2012;68:1563-69 CrossRef Medline

13. Hopp K, Popescu BF, McCrea RP, et al. Brain iron detected by SWI high pass filtered phase calibrated with synchrotron X-ray fluorescence. J Magn Reson Imaging 2010;31:1346-54 CrossRef Medline

14. Wiggermann V, Hametner S, Hernandez-Torres E, et al. Susceptibility-sensitive MRI of multiple sclerosis lesions and the impact of normal-appearing white matter changes. NMR Biomed 2017;30 CrossRef Medline

15. Eskreis-Winkler S, Deh K, Gupta A, et al. Multiple sclerosis lesion geometry in quantitative susceptibility mapping (QSM) and phase imaging. J Magn Reson Imaging 2015;42:224-29 CrossRef Medline

16. Wang Y, Liu T. Quantitative susceptibility mapping (QSM): decoding MRI data for a tissue magnetic biomarker. Magn Reson Med 2015;73:82-101 CrossRef Medline

17. Wisnieff C, Ramanan S, Olesik J, et al. Quantitative susceptibility mapping (QSM) of white matter multiple sclerosis lesions: interpreting positive susceptibility and the presence of iron. Magn Reson Med 2015;74:564-70 CrossRef Medline

18. Stuber C, Pitt D, Wang Y. Iron in multiple sclerosis and its noninvasive imaging with quantitative susceptibility mapping. Int $\mathrm{J} \mathrm{Mol}$ Sci 2016;17:E100 CrossRef Medline

19. Deistung A, Schäfer A, Schweser F, et al. Toward in vivo histology: a comparison of quantitative susceptibility mapping (QSM) with magnitude-, phase-, and $\mathrm{R} 2^{*}$-imaging at ultra-high magnetic field strength. Neuroimage 2013;65:299-314 CrossRef Medline

20. Langkammer C, Liu T, Khalil M, et al. Quantitative susceptibility mapping in multiple sclerosis. Radiology 2013;267:551-59 CrossRef Medline

21. Zhang Y, Gauthier SA, Gupta A, et al. Quantitative susceptibility mapping and $\mathrm{R} 2^{*}$ measured changes during white matter lesion development in multiple sclerosis: myelin breakdown, myelin debris degradation and removal, and iron accumulation. AJNR Am J Neuroradiol 2016;37:1629-35 CrossRef Medline

22. Li X, Harrison DM, Liu H, et al. Magnetic susceptibility contrast variations in multiple sclerosis lesions. J Magn Reson Imaging 2016; 43:463-73 CrossRef Medline

23. Harrison DM, Li X, Liu H, et al. Lesion heterogeneity on high-field susceptibility MRI is associated with multiple sclerosis severity. AJNR Am J Neuroradiol 2016;37:1447-53 CrossRef Medline

24. Nguyen TD, Wisnieff C, Cooper MA, et al. T2 prep three-dimensional spiral imaging with efficient whole brain coverage for myelin water quantification at 1.5 Tesla. Magn Reson Med 2012;67:614-21 CrossRef Medline

25. Nguyen TD, Deh K, Monohan E, et al. Feasibility and reproducibility of whole brain myelin water mapping in $\mathbf{4}$ minutes using fast acquisition with spiral trajectory and adiabatic T2prep (FAST-T2) at 3T. Magn Reson Med 2016;76:456-65 CrossRef Medline

26. Laule C, Kozlowski P, Leung E, et al. Myelin water imaging of multiple sclerosis at $7 \mathrm{~T}$ : correlations with histopathology. Neuroimage 2008;40:1575-80 CrossRef Medline

27. McCreary CR, Bjarnason TA, Skihar V, et al. Multiexponential T2 and magnetization transfer MRI of demyelination and remyelination in murine spinal cord. Neuroimage 2009;45:1173-82 CrossRef Medline

28. Vargas WS, Monohan E, Pandya S, et al. Measuring longitudinal 
myelin water fraction in new multiple sclerosis lesions. Neuroimage Clin 2015;9:369-75 CrossRef Medline

29. de Rochefort L, Liu T, Kressler B, et al. Quantitative susceptibility map reconstruction from MR phase data using Bayesian regularization: validation and application to brain imaging. Magn Reson Med 2010;63:194-206 CrossRef Medline

30. Liu J, Liu T, de Rochefort L, et al. Morphology enabled dipole inversion for quantitative susceptibility mapping using structural consistency between the magnitude image and the susceptibility map. Neuroimage 2012;59:2560-68 CrossRef Medline

31. Jenkinson M, Bannister P, Brady M, et al. Improved optimization for the robust and accurate linear registration and motion correction of brain images. Neuromage 2002;17:825-41 CrossRef Medline

32. Chen W, Gauthier SA, Gupta A, et al. Quantitative susceptibility mapping of multiple sclerosis lesions at various ages. Radiology 2014;271:183-92 CrossRef Medline

33. Zhang Y, Gauthier SA, Gupta A, et al. Longitudinal change in magnetic susceptibility of new enhanced multiple sclerosis (MS) lesions measured on serial quantitative susceptibility mapping (QSM). J Magn Reson Imaging 2016;44:426-32 CrossRef Medline
34. Kuhlmann T, Ludwin S, Prat A, et al. An updated histological classification system for multiple sclerosis lesions. Acta Neuropathol 2017;133:13-24 CrossRef Medline

35. Wiggermann V, Hernández Torres E, Vavasour IM, et al. Magnetic resonance frequency shifts during acute MS lesion formation. $\mathrm{Neu}$ rology 2013;81:211-18 CrossRef Medline

36. Haider L. Inflammation, iron, energy failure, and oxidative stress in the pathogenesis of multiple sclerosis. Oxid Med Cell Longev 2015; 2015:725370 CrossRef Medline

37. Haider L, Fischer MT, Frischer JM, et al. Oxidative damage in multiple sclerosis lesions. Brain 2011;134:1914-24 CrossRef Medline

38. Prineas JW, Kwon EE, Cho ES, et al. Immunopathology of secondary-progressive multiple sclerosis. Ann Neurol 2001;50:646-57 CrossRef Medline

39. Cronin MJ, Wharton S, Al-Radaideh A, et al. A comparison of phase imaging and quantitative susceptibility mapping in the imaging of multiple sclerosis lesions at ultrahigh field. MAGMA 2016;29: 543-57 CrossRef Medline 